\title{
Research on the Characteristics and Communication Mode of Interesting Virtual Community: Take Net Ease Cloud Music as an Example
}

\author{
Xiaolin Chen \\ ${ }^{1}$ Center for Digitalized Culture and Media, University of Electronic Science and Technology of China, \\ Chengdu, China; \\ ${ }^{2}$ School of Public Administration, University of Electronic Science and Technology of China, \\ Chengdu, China. \\ cxlcxlchen@163.com
}

Keywords: music community, virtual community, Net ease Cloud Music, communication mode.

\begin{abstract}
With the rapid development of the Internet, the number of music users in the mobile phone network is increasing, and the ferocious development of various music platforms deserves attention. At present, domestic research on music virtual communities is very lacking. This article takes the virtual community as the starting point and selects the unique Net ease Cloud Music community as its main research object. Starting from the characteristics of the Net ease Cloud Music community, the defines the members of the Net ease Cloud Music community and analyzes the characteristics of different members and the characteristics of UGC content products. The study of the communication model of the Net ease Cloud Music community starts with the main body, the structure, and the scope of communication, and explores the multi-level compound communication model and characteristics of decentralized communication the Net ease Cloud Music community.
\end{abstract}

\section{Introduction}

According to the 41 st China Internet network development state statistic report, as of December 2017, the number of online music users reached 548 million, an increase of 44.96 million compared with the end of last year, accounting for $71.0 \%$ of the total Internet users. The number of mobile network music users reached 512 million, an increase of 43.81 million from the end of last year, accounting for $68.0 \%$ of mobile Internet users. It is the fourth highest frequency for Internet users to use mobile phones to use software. The utilization rate of netizens is as high as $68.0 \%$, which represents an annual growth rate of 9.4\% year-on-year at the end of last year [1]. The mobile phone music market is prosperous and various digital music platforms are exploring to create distinctive market positioning. For example, KuGou music platform and QQ music platform focusing on fan of the economy, trying to make the layout from the music IP to star IP, trying to the use of celebrity influence to maintain user and platform stickiness. Net ease Cloud Music explores new paths, building emotional support network, playing with the user's creativity to create a thread culture, and with the professional musicians joining and friends' pushes. Singing, DJ programming, social networking, and geographical location as core elements to discover and share music to build online virtual music social ecosystem.

\section{Virtual Community}

People in the virtual community use computer screen to communicate, to exchange pleasures and arguments, to engage in the exchange of knowledge, business, share emotions, make plans, brainstorm, gossip, make friends and create art. With the development of computer science and technology, the form of online virtual communities is not static. There is no unified definition of a virtual community. Different perspectives can be used to interpret and define the virtual community differently. In order to emphasize the perspective of participant relationship in the community, the definition of virtual community proposed by $\mathrm{H}$. Rheingold (1993) is the earliest and has been widely 
recognized and quoted. He believes that the virtual community is a social group that forms a network of people in cyberspace after a long period of time when many people have discussed and generated strong enough emotions on the Internet. [2] Domestic scholars Xu Xiaolong and Wang Fanghua (2007) believe that virtual communities are the sum of group relationships with common goals that people form in order to meet certain needs and interact with each other in cyberspace. [3]

There are various topics and forms of virtual communities emerging on the Internet. There is no universally accepted standard for the classification of virtual communities. This article selects interested virtual communities in virtual communities for research. The method of division is from foreign scholars. Hagel and Amstrong, they proposed to divide the virtual community into four types: interest type, relationship type, fantasy type, and transaction type. [4] Among them, the interest type virtual community is gathered by people with common interests and hobbies to share and discuss exchanges. community. The Net ease Cloud Music community is based on the theme of music in the Internet and has assembled a group of people who love music and want to learn about music and provide them with a virtual community of mutual interest, sharing, entertainment and entertainment.

\section{The Features of Net Ease Cloud Music Community}

\subsection{Net Ease Cloud Music ---- Creative Music Production Community.}

Net ease Cloud Music Community is a creative production community. UGC's production model has enabled community members to create song lists, foreign language lyrics, comments, and DJ-made programs. They can choose one of the factors and integrate them into a song list with corresponding specific meanings, themes based on the different criteria of the song's different styles, moods, styles, genres, and comments. For the translation of lyrics, the solid translation model is out of date, they find a new way to embody the emotions and integrate into the background knowledge of the singers. Particularly, in the translation of rap songs, the rap rhyme and implied meanings of the lyrics need to understand both the cultural differences and professional terms of the two countries, then can penetrate the songs the singer wants to express. Net ease Cloud Music's song reviews created a trend of listening music right after seeing comments. Unique song commentary made the music into a new story. It was often such a few words that hit many members of the community, whether it was sad or cheerful. The melody itself can arouse their empathy and make the song itself more story-like and inflammatory. According to the type of songs the community members listen to, the collection of song genre, and personal preferences which are based on sophisticated algorithms to accurately push DJ home-made programs.

\subsection{Features of Net Ease Cloud Music Production Community Members.}

Members of the Net ease Cloud Music Community can be divided into visitors, socializers, and content producers based on the degree of production of community content products.

Visitors means not registered in the Net ease Cloud Music Community or have registered but did not made any content producers. Visitors pay attention to the community's content that is not stereotyped or pan-topic information, they search for the specific part of their own needs, almost no participation in any comments and interactions.

The socializer refers to the registered members who are registered in the community and actively communicates, shares, or hit likes to other members. They can establish relationships with others and frequently active in the community. They are an integral part of enhancing community stickiness. The Net ease music community has created a dynamic model in the friend interface, a dynamic circle that can share songs, pictures and text. Active socializers use pictures and texts to express their cognition and preference for music. Musical personalities are more three-dimensional. Discovering high-quality music can also send it directly to designated friends for sharing and sharing while the music is being released.

Producers are an important part of the Net ease Cloud Music community. They come from ordinary members, professional DJs, musicians. They created high-quality reviews that appealed to the public, created explosive songs and radio stations, and translated gripping foreign language lyrics. These 
scattered and independent heterogeneous individuals built a community and a specific collective circle with common interests through the interactive platform of the Internet and Net ease Cloud Music. Allow individuals to interact and links with platform content in the collective common circle. According to data from the Net ease music community, more than half of the community members have established a habit of listening to songs, viewing comments, sharing, and publish song lists. The creation of UGC quality playlists and music reviews can be subdivided and community members, and groups of diverse groups are gathered to enhance community cohesiveness.

\subsubsection{Net Ease Cloud Music Community Members Have Spontaneity.}

Whether it is from the perspectives of social exchange theory, social cognitive theory, social capital theory, expectation value theory, planned behavior theory, technology acceptance model, organizational citizen behavior theory, social sense and relationship face, etc. Analysis of influencing factors of virtual community knowledge sharing from the perspective of existing theory, whether for any expectation or purpose, is spontaneous. Musicians upload music, DJ homemade programs, song reviews and translations are the author's self-cognition of the contemporary mainstream consciousness. According to their different quality background, when faced with the same music material is the same light refraction to the polyhedron, will present a different refraction different point of view, makes individuality creation show the rich and colorful forms. From the perspective of Stephenson's game theory, the members who created the songs of the Net ease Cloud Music Community repeatedly, are not so much a tool to express emotions as the Net ease Cloud Music Community. It is for playing. In the comments, they constantly responded and hit likes out of play. Therefore, they participate in the Net ease Cloud Music community activities can be assumed that based on the purpose of the game. Communication in games is not purposeful. It is just to find fun or to express emotions. It is always pleasant for the community members.

Net ease Cloud Music community song list. Using music as emotional carrier causes emotional resonance. If you like the same song list, it is the resonance between the song list creator and the listener and the resonance between the listeners. It is a community gathering point for community members, with a point of connection and a line. It is convenient for the social identity to provide content between the weak links, and such content is created by the community members themselves, so the song list gives a deeper meaning.

Net ease Cloud Music Community song reviews. The maximum number of words for each song is 140 words. When the number of songs is accumulated to more than 999, the "999+" logo will appear on the interface of the single song. This is often used as a measure of how popular a song is. Simple and easy-to-understand community rules and low participation thresholds help to increase member enthusiasm and participation.

\subsubsection{Producers Have Individuality and a Combination of Virtual and Real Identity.}

The combination of virtual and real identity is also the formation of strong relationship chains and weak relationship chains. Songs, popular reviews, DJ radio and other music and cultural activities are always based on individual production. Net ease Cloud Music Community has a variety of registration forms: WeChat, QQ, Sina Weibo, Net ease E-mail and mobile phone numbers can all be registered. You can choose whether to use a virtual identity or real one to register and login. Using social accounts with friends and relatives to $\log$ in, you can find acquaintances who are playing in the community at the first time, so that they can identify each other and add links to strengthen the relationship chain. If you use a virtual identity to join a community, individuals can bury their identity and social attributes in real life. Presenting in a virtual community is a name or character. Its anonymity and physical absence make it out of the social attributes of reality and exist. Virtual users can only use the add friend to view the other party's dynamics, song list, song list and personal basic information; this part belongs to the weak relationship in the interpersonal relationship network. Granovetter's Weak Relationship Theory believes that weak relationships can speed up the flow of information between different groups and groups. Weak-relationship groups have a wide range of characteristics and are not rigidly tied to a group that is related to themselves. They do not exclude 
foreign cultures. Different viewpoints can be penetrated through weaker relationship media from other groups.

\subsection{Net Ease Cloud Music Community Content Product Productivity and Creativity.}

The products produced by Net ease Cloud Music, such as song lists, lyrics, reviews, DJ's home-made programs, music uploaded by musicians are creative. The combination of song commentary storytelling and sensational as well as various songbooks can show the creativity of the results, in which the emotions expressed have universal, simple, typical emotional words that can move and infect more people. The creativity of community products is also fully reflected in the design concept of song list. That is, the uniqueness of the theme of the song list and the content of the response. For example, there will be different types of songs, different purposes, different uses, different styles of songs in the popular song list:

Some of the listed hit lists have integrated unrelated songs to include theme music in specific scenes, such as sports, reading and cafes, popular videos, underground music, live music, practical purpose value music, and idol singers. Singing collections, song combinations with certain common characteristics, and knowledge learning. These songs manifested their uniqueness in the process of being collected, played, shared, and commented. Creative product achievements are the essence of the creation of production by the Net ease Cloud Music community. These creation and production products are examples of community activity and vitality.

Unlike ordinary commodity production, the products of the music community are not material basic products that meet the needs of the people for food, clothing, housing, and transportation, but are relative to the production form-creative spiritual products that combine the spiritual needs of the broad masses of the people. People choose their own production positioning based on their own background, interest, pursuit, taste and other factors. Spiritual products are objectively commodity [5], so the Net ease Cloud Music community content products are commodity-oriented.

\section{Net Ease Cloud Music Transmission Mode Analysis}

The communication environment of the virtual community first ensures the uniqueness of the way of community communication. Net ease Cloud Music community communication is different from any traditional communication model. The openness and interaction of virtual community make the relationship between the communicator and the audience present a trend of integration and decentralization. For the dissemination of information, communicator transmission and audience acceptance are mutually dependent, and better communication effects can be achieved in a good interaction. In the virtual community, information publishing presents a variety of features. The Net ease Cloud Music community has the characteristics of independence, immediacy, and interaction. The equitable communication model broadens the communication channels and integrates interpersonal relationships.

The multi-level compound communication mode can be transmitted between members of the community through short messages. The content producer can realize multi-center and one-to-many communication and fission communication of mesh connections through comments and song lists outside the community. Members upload music with a community logo or share music reviews to social media such as WeChat, Weibo, etc. In which various behaviors such as retransmission and commentary are information feedback, so that the secondary creative communication based on the community forms a secondary spread. Mass communication and interpersonal communication are carried out through it, forming a multi-level mode of compound communication.

Decentralized communication, the traditional mode of communication is carried out from the top down, and the right to communication has always been in the hands of authority. In the Net ease Cloud Music Virtual Community, a network-based communication platform, community members can have dual identities at the same time, that is, the transmitter and the audience. Net ease Cloud Music community has gradually formed a more mature music social closed-loop, to the joint construction of the spread of the role. Breaking the authoritative position and characteristics of 
traditional center communication mode. Community members are not merely information receivers, but also can become information disseminators. The equitable communication opportunities in the Net ease Cloud Music community have driven information interaction beyond the limitations of time and space, making it impossible for communicators and audiences to interact and feedback channels.

\section{Conclusion}

Net ease Cloud Music community is a real-time interactive media platform. The community content products meet the needs of member fragmentation and scene-based recreation and entertainment. Personalized recommendation that focuses on data analysis can accurately push music, multi-level compound mode of communication, and promote community development. And it has played a very important role in enhancing community cohesion.

\section{References}

[1]. 41st China Internet network development state statistical report

[2]. Rheingold H.Cyburgs. Book Review: The Virtual Community. Homesteading on the Electronic Frontier [J]. Science, 1994, 265(5157):1114.

[3]. Xiao long $\mathrm{Cu}$, Fang Hua Wang. Research on the Frontier of Virtual Community Research. Foreign economy and management.2007(9):10-16

[4]. Hagel J. Net gain. Expanding Markets Through Virtual Communities. Journal of Interactive Marketing, 1999, 13(1): 55-65.

[5]. Siangtan Li. The commodication of Mental Products. Academic Research. 1985(5):44-48. 